\title{
Abnormal expression of IncRNA UCA1 disturbed cell apoptosis through mediating mitochondrial dynamics in PDAC
}

\author{
BuWei TENG, Tao FENG, Wei LI, Zhong WANG* \\ The First People's Hospital of Lianyungang, Lianyungang Clinical College of Nanjing Medical University, Lianyungang, Jiangsu, China \\ *Correspondence: lygwangzhong@126.com
}

Received June 29, 2020 / Accepted November 25, 2020

\begin{abstract}
A great number of studies have shown the pivotal role of mitochondria in cancer progression and numerous studies indicated that lncRNAs are involved in tumor metabolism. However, the relationship between UCA1 and mitochondria in PDAC remains unclear. Here, we reported for the first time that UCA1-driven change in mitochondrial dynamics induced mitochondrial apoptotic pathway in PDAC. In this research, data mining revealed that upregulated UCA1 occurred in PDAC patients, which meant a high likelihood of a poor prognosis. Following, UCA1 silencing could notably decrease cell viability and induce cell apoptosis. Further research revealed that UCA1 silencing could induce more cytochrome clocalization in the cytosol, which triggered the mitochondrial apoptotic pathway in PDAC cell lines. Meanwhile, the morphological analysis showed significantly enhanced mitochondrial fragmentation presented in UCA1 knockdown cells, coupled with increased expression of Drp1 and Fis1, together with an activation form of Drp1, which would promote mitochondria fission. Additional, mitochondrial fission inhibitor Mdivil markedly reversed the effects of the UCA1 knockdown on cell apoptosis in PDAC. Collectively, we deduce that UCA1-driven change in mitochondrial dynamics induced the mitochondrial apoptotic pathway in PDAC. Therefore, lncRNA UCA1 could be considered as a promising therapeutic target for the poor prognosis in PDAC.
\end{abstract}

Key words: long non-coding RNA UCA1, pancreatic ductal adenocarcinoma, mitochondrial dynamics, mitochondrial apoptotic pathway

Pancreatic ductal adenocarcinoma (PDAC), a common malignancy of the digestive tract, possesses a high fatality rate worldwide with a 5-year survival of less than $5 \%[1]$. Despite the great advancement of diagnosis and treatment has been made, most of the PDAC patients are difficult to be diagnosed at early stages due to the lack of obvious symptoms and effective early detection methods [2]. Therefore, more efforts should be paid to elucidate tumorigenesis. And novel strategies for early detection and treatment are urgent to be developed.

Long non-coding RNAs (lncRNAs), a group of RNAs (> $200 \mathrm{nt}$ ) with no coding capacity due to an incomplete open reading frame [3], have been reported to exert a role in multiple biological processes, including chromatin modification [4], X-chromosome inactivation [5], microRNA sponging [6], etc. Accumulating evidence has linked dysregulation of lncRNAs with various malignant tumors [7], including urothelial carcinoma associated 1 (UCA1). In breast cancer, the $\mathrm{Wnt} / \beta$-catenin signaling pathway was active to promote EMT by UCA1 [8]. In PDAC, upregu- lated UCA1 promotes tumorigenesis via regulating the Hippo pathway [9]. In bladder cancer, UCA1 is involved in regulating the Warburg effect through the mTOR/STAT3/ miR-143/HK2 signaling pathway [10]. Moreover, UCA1 could enhance mitochondrial function through the UCA1/ miR-195/ARL2 axis in bladder cancer [11]. However, few studies were carried out to explore whether UCA1 participates in mitochondrial function in PDAC.

In this study, we investigated the roles and the mechanism undergoing in the PDAC progress of UCA1. As well, the current study provides the first evidence that UCA1-driven change in mitochondrial dynamics induced the mitochondrial apoptotic pathway in PDAC.

\section{Materials and methods}

Data mining. The Kaplan-Meier plotter, an online analysis tool, was used to analyze the relationship between UCA1 expression and patients' outcomes (http://kmplot.com/ analysis/). Log-rank p-values were calculated and displayed. 
The GEO (GSE16515) and TCGA datasets were used in this study to analyze UCA1 in PDAC. The GEO datasets can be found at https://www.ncbi.nlm.nih.gov/geo.

Cell culture. Human PDAC cell lines and the non-malignant hTERT-HPNE (HPNE) cells were cultured in a complete DMED medium with $10 \% \mathrm{FBS}$ at $37^{\circ} \mathrm{C}$ with $5 \% \mathrm{CO}_{2}$. ASPC1 and SW1990 were transfected with lentivirus (lentivirus-shUCA1 or empty vehicle control) in the presence of $8 \mu \mathrm{g} / \mathrm{ml}$ polybrene (Sigma) and screened with $2 \mu \mathrm{g} / \mathrm{ml}$ puromycin (Sangon, Shanghai) for 2 weeks.

Real-time PCR. Total RNA was isolated using RNAiso Plus (Takara) according to the manufacturer's protocol. cDNA was synthesized using the RevertAid First Strand cDNA Synthesis kit (Takara). The mRNA expression was measured by using an SYBR Green qPCR assay (Roche). Primers for the qRT-PCR were as follows: U6 primer forward, 5'-CTCGCTTCGGCAGCACA-3' and reverse, 5'-AACGCTTCACGAATTTGCGT-3'; UCA1 primer forward, 5'-TTTGCCAGCCTCAGCTTAAT-3' and reverse, 5'-TTGTCCCCATTTTCCATCAT-3'. The $2^{-\Delta \mathrm{Ct}}$ method was used to calculate the relative levels of gene expression.

Cell transfection. Lentiviral vector expressing shRNAUCA1 and the corresponding negative control shRNA-NC were purchased from GeneChem (Shanghai, China). Cell infection was performed following the manufacturer's protocol. Cells stably transfected with shRNA-UCA1 or shRNA-NC were selected by puromycin $(2 \mu \mathrm{g} / \mathrm{ml})$. The shRNA sequence as shown below: shRNA-UCA1-1 Sense: GUUAAUCCAGGAGACAAAGATT, Antisense: UCUUUGUCUCCUGGAUUAACTT; shRNA-UCA1-2 Sense: GGACAACAGUACACGCAUAdTdT, Antisense: TATGCGTGTACTGTTGTCCdTdT.

CCK-8 assay. 96-well plates were seeded with $5 \times 10^{3}$ cells/well $(100 \mu \mathrm{l} /$ well $)$ and cultured at $37^{\circ} \mathrm{C}$. The absorbance at $450 \mathrm{~nm}$ was monitored with $10 \mu \mathrm{l}$ Cell Counting Kit-8 (CCK-8, WST-8, Dojindo, Japan) per well using a microplate reader. The experiment was 3 times repeated.

Western blot analysis. Western blot studies were performed as previous describe [12]. The primary antibodies: Bax (ab32503, Abcam), Bcl2 (2876, CST), Bcl-xl (2762, CST), Drp1 (8570, CST), Fis1 (sc-98900, Santa), cytochrome c (ab133504, Abcam), cleaved Caspase-3 (ab2302, Abcam), cleaved Caspase-9 (52873, CST), COX-IV (MA5-15078, Thermo), and $\alpha$-tubulin (ab7291, Abcam).

Annexin V/PI staining assay. This assay was performed as previously reported [13].

Caspase activity assay. Caspase Activity Assay Kit (Beyotime, China) was used to measure caspase-3 and caspase- 9 activities in accordance with the manufacturer's protocol.

Mitochondrial morphometrics. MitoTracker Red (Invitrogen) was used to stain mitochondria according to the manufacturer's instruction. A laser scanning confocal microscope was used to acquire images. Mitochondrial morphology was quantified as described before [14].
Statistical analysis. GraphPad 7.0 was used to carry out statistical analysis by Student's $t$ test. Cumulative survival time was calculated by the Kaplan-Meier method. A p-value $<0.05$ was considered as statistically significant.

\section{Results}

Aberrant overexpression of UCA1 predicted poor prognosis in PDAC patients. As a first attempt to identify the relationship between UCA1 and prognosis in multiple common digestive tract tumors, including esophagus adenocarcinoma, esophagus squamous cell carcinoma, liver hepatocellular carcinoma, pancreatic ductal adenocarcinoma (PDAC), rectum adenocarcinoma, and stomach adenocarcinoma, we conducted analysis utilizing the online Kaplan Meier plotter (http://kmplot.com/analysis/). Interestingly, statistically significant only existed in PDAC about UCA1 expression and prognostic, when set the mRNA expression median as a cutoff. As shown in Figure 1A, p-value was $<0.05$ in UCA1 expression and overall survival (OS), as well as in UCA1 expression and relapse-free survival (RFS). Further analysis showed that poor OS and median survival time (18.93 vs. 24.46 months) existed in PDAC patients with high UCA1 expression (log-rank p=0.014, Figure 1B). Similar trend existed in UCA1 expression and RFS (log-rank $\mathrm{p}=0.023,12.6$ vs. 32.9 months, Figure 1C). Meanwhile, we verified that high UCA1 expression was strongly correlated with poor OS in TCGA (Figure 1D). Following, data mining in GSE16515 showed the top 20 lncRNAs, which were abnormally increased in PDAC tissues and UCA1 was one of the upregulated lncRNAs (Figure 1E). Similarly, notably higher UCA1 expression was verified in pancreatic tumor tissues than in normal tissues $(\mathrm{p}<0.001$, Figure $1 F)$. And, dramatically upregulated UCA1 expression was observed in multiple PDAC cell lines (PANC1, Capan1, SW1990, Capan2, ASPC1, and HPAC) compared with that in the nonmalignant hTERTHPNE (HPNE) cells (Figure 1G).

UCA1 silencing could suppress cell proliferation. To determine the role of UCA1 on PDAC cell viability, ASPC1 and SW1990 cells were selected to transfect with shRNA-UCA1 (Figures 2A, 2B), respectively. The CCK-8 assay showed that UCA1 silencing significantly inhibited cell viability rates in ASPC1 (Figure 2C) and SW 1990 (Figure 2D) cells, compared to the control cells. What's more, a similar phenomenon was shown by the colony formation assay (Figures 2E, 2F).

UCA1 silencing induced cell apoptosis in PDAC. The process of cell apoptosis was involved in tumor progression, including PDAC. The results of the gene set enrichment analysis (GSEA) indicated that high UCA1 expression was positively correlated with PDAC (Figure 3A) and cell apoptosis (Figure 3B). Hence, we conducted the Annexin V-PI assay to analyze the cells' apoptosis with UCA1 knockdown or without. The results showed that UCA1 knockdown resulted in a significant increase of cell apoptosis in ASPC1 and SW1990 cells, compared to the control cells (Figures 3C, 


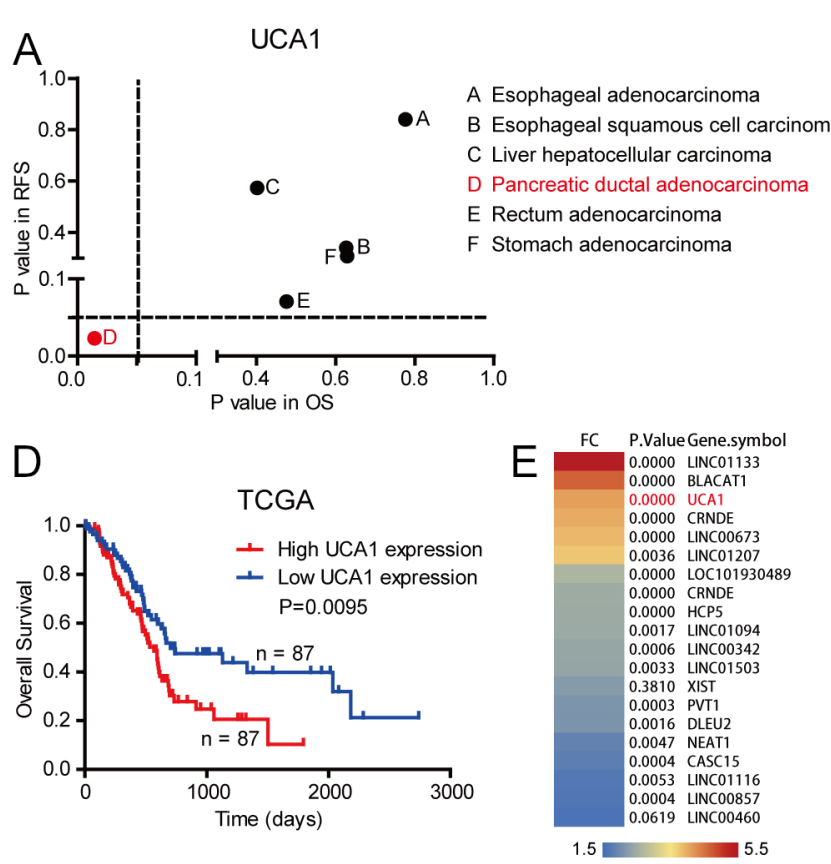

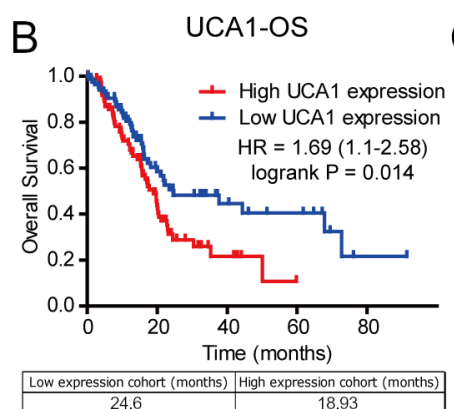

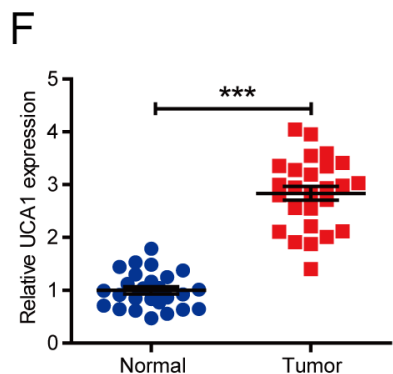

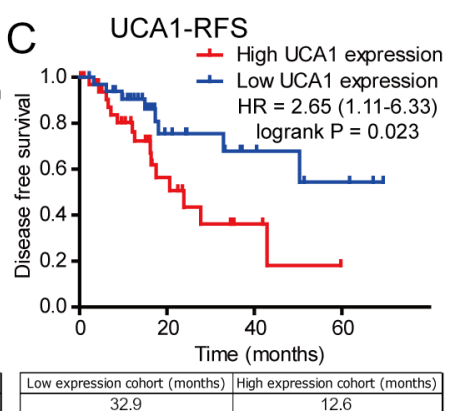

G

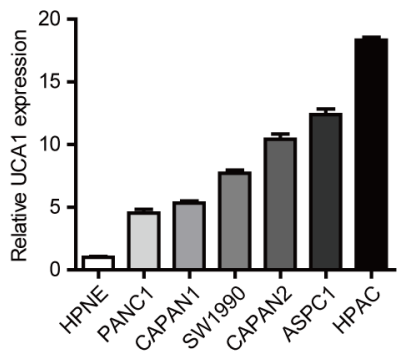

Figure 1. Upregulated UCA1 expression predicted the poor prognosis in PDACs. A) The p-value distribution of OS and RFS about lncRNA UCA1 expression in multiple common digestive tract tumors analyzed by Kaplan Meier plotter (http://kmplot.com/analysis/). B) Kaplan-Meier plotter showed poor OS and median survival time existed in PDAC patients with high IncRNA UCA1 expression (log-rank p=0.014, HR=1.69). C) Kaplan-Meier plotter showed poor RFS and median survival time existed in PDAC patients with high lncRNA UCA1 expression (log-rank $p=0.023$, HR=2.65). D) Kaplan-Meier OS curves in the TCGA dataset of PDAC patients. Patients were grouped based on the median expression value of UCA1 expression. E) Heatmap showed UCA1 was the IncRNA with relatively large expression changes in GSE16515. F) Analysis of UCA1 mRNA levels in paired PDAC and non-tumor tissues $\left(n=26,{ }^{* *} p<0.001\right)$. G) qRT-PCR was used to detect the relative UCA1 expression in PDAC cell lines and the non-malignant hTERT-HPNE (HPNE) cells.
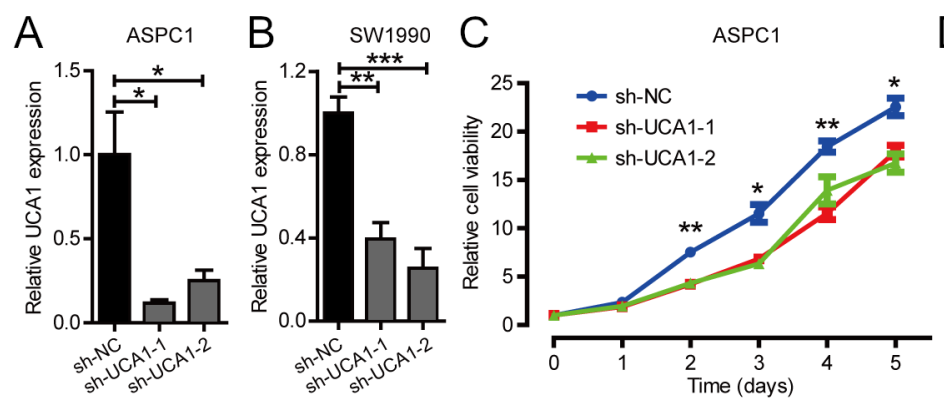

D

SW1990
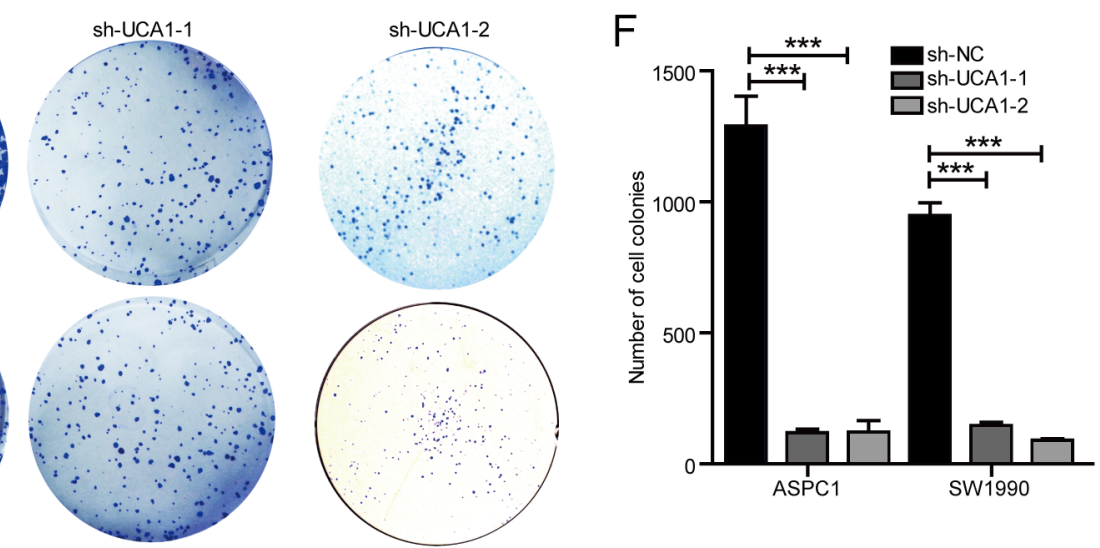

Figure 2. UCA1 silencing decreased cell viability. A, B) ASPC1 and SW1990 cells were selected to be transfected with sh-UCA1-1 and sh-UCA1-2. C, D) UCA1 silencing markedly inhibited cell viability both in ASPC1 (C) and Canpan1 (D) cells. E, F) UCA1 knockdown significantly inhibited the clone formation capacity of PDAC cells. 
3D). Moreover, higher caspase-3 (Figure 3E) and caspase-9 (Figure 3F) activities in UCA1-knocked down cells were detected. Meanwhile, higher cleaved forms of caspase-3 and caspase-9 were detected in UCA1-knocked down cells (Figure 4A). Herein, we concluded that UCA1 silencing induced cell apoptosis in PDAC.
UCA1 knockdown induced the mitochondrial apoptotic pathway. Following, we detected the biomarkers of mitochondrial apoptosis pathway after UCA1 knockdown. We found that UCA1 knockdown led to a significant increase of pro-apoptotic markers (BAX and PARPI), as well as a decrease in anti-apoptotic markers (Bcl-2 and BclxL;
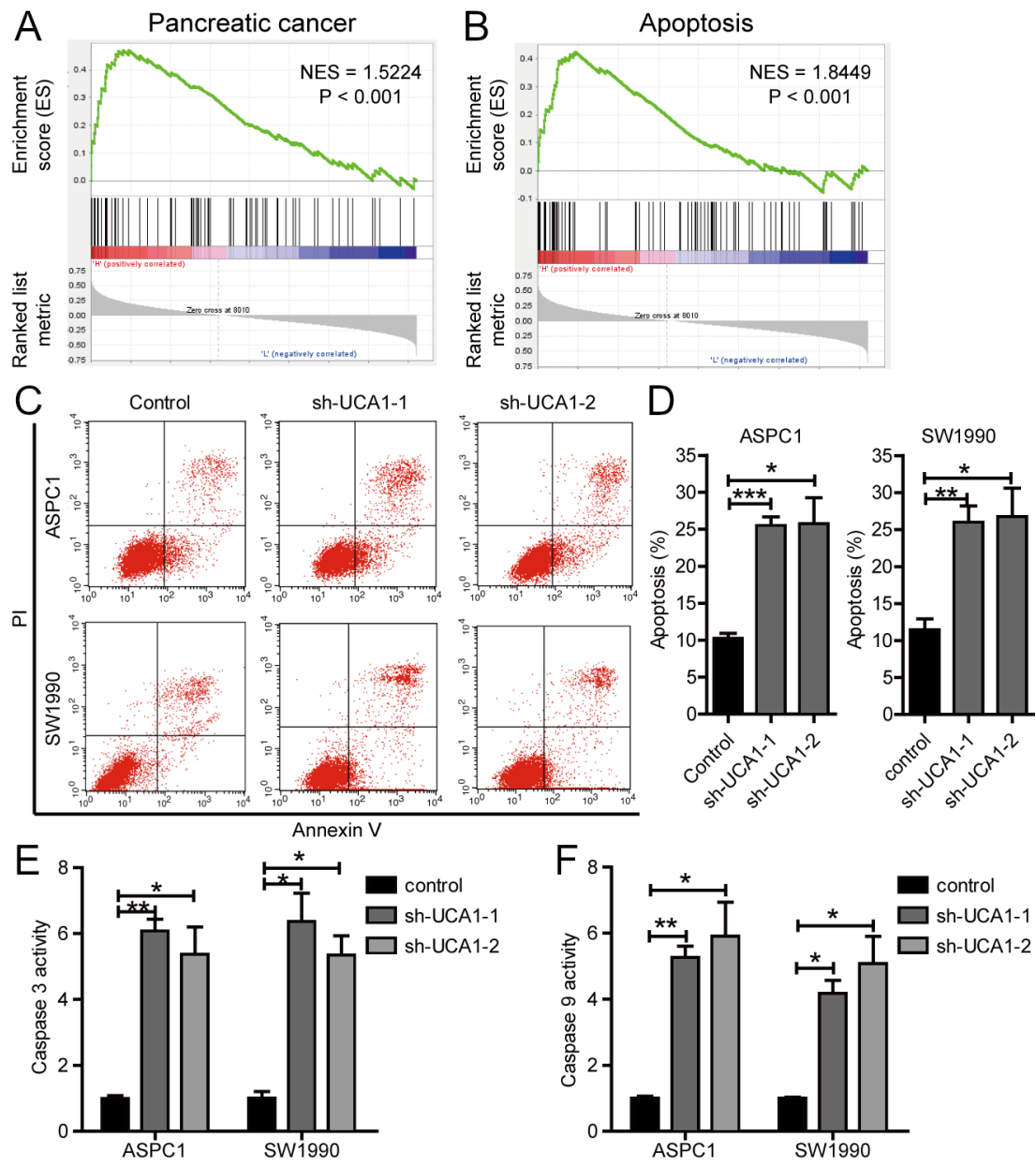

Figure 3. UCA1 silencing induced apoptosis in PDAC cell lines. A, B) Gene enrichment analysis based on the UCA1 expression from the TCGA dataset. NES, normalized enrichment score. C) UCA1 knockdown induced apoptosis in PDAC cells stained with Annexin V-APC/PI as analyzed by flow cytometry. D) The graph showed the percentage of apoptosis for each treatment group. Student's t-test, ${ }^{* * *} p<0.001,{ }^{* *} p<0.01$. E, F) UCA1 knockdown enhanced caspase-3 (E) and caspase-9 (F) activity in PDAC cells, compared to the control group. ${ }^{*} \mathrm{p}<0.05,{ }^{* *} \mathrm{p}<0.01$
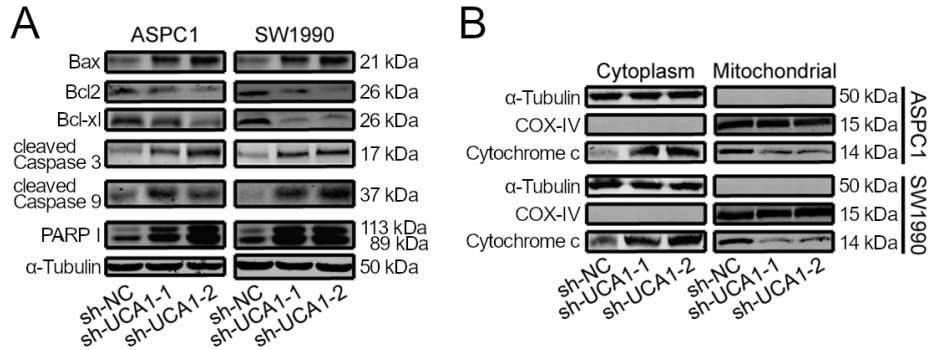

Figure 4. UCA1 knockdown induced the mitochondrial apoptotic pathway. A) The mitochondrial apoptotic markers: BAX, Bcl2, Bcl-xl cleaved caspase 3, cleaved caspase 9, and PARPI were assessed in PDAC cells B) UCA1 knockdown altered the subcellular distribution of cytochrome c in the cytoplasm and the mitochondrial fraction of PDAC cells. $\alpha$-tubulin and COX-IV were used as a loading control for cytosol and mitochondria, respectively. 
Figure 4A). What's more, the UCA1 knockdown resulted in a marked increase of cytochrome $c$ release into cytosolic fractions, corresponding to the decrease in mitochondrial fractions (Figure 4B). These results indicated that UCA1 knockdown is involved in the mitochondrial apoptotic pathway.

UCA1 silencing enhanced mitochondrial fission in PDAC cells. Considering that the releasing of cytochrome c from mitochondria may be a result of mitochondrial fission [15], we studied how UCA1 affected mitochondrial dynamics. With that in mind, we assessed the role of UCA1 on mitochondrial fission. The results showed that UCA1 silencing led to a significantly enhanced mitochondrial fragmentation, which was accompanied by a decrease in mitochondrial reticulation (Figures 5A, 5B). Following that, we measured the change of mitochondrial fission and fusion markers by immunoblotting. We discover that significantly increased Drp1 and Fis1 expression, coupled with increased
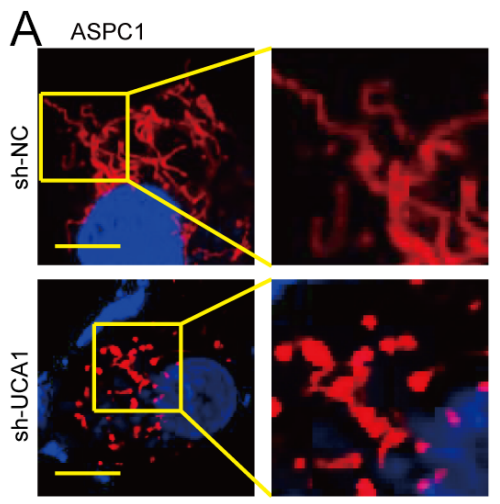

Mito Tracker Red Hoechst 33342
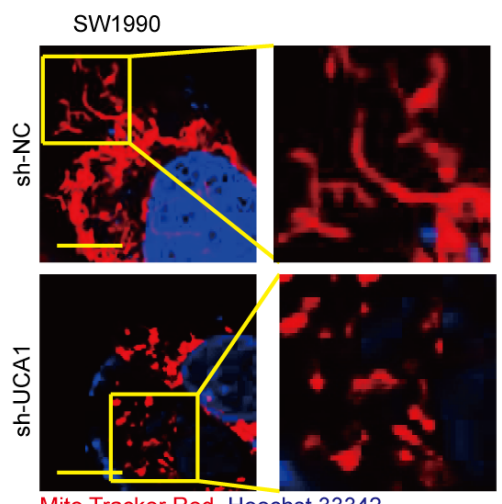

Mito Tracker Red Hoechst 33342
D

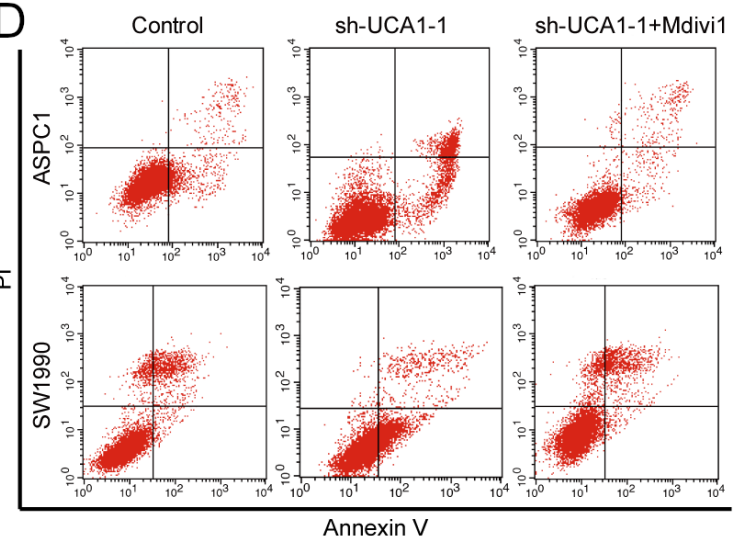

B
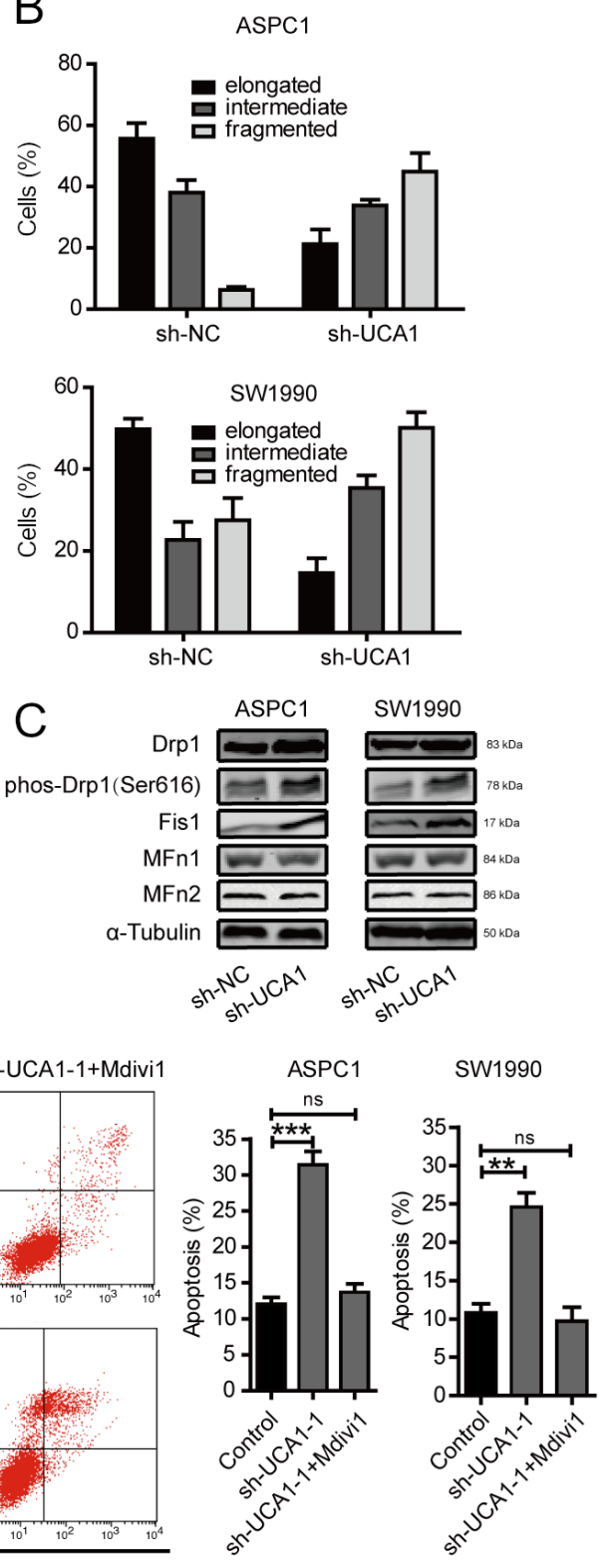

Figure 5. UCA1 silencing enhanced mitochondrial fission. A) More mitochondrial fragments were present in UCA1 knockdown cells. Cells were stained with Mito-Tracker Red (red) and Hoechst33342 (blue). B) Histogram of mitochondrial morphology reflected the degree of mitochondrial morphological change in cells ( $\mathrm{n}=100)$ with different treatments. C) Mitochondrial fission and fusion markers (Drp1, phos-Drp1, Fis1, MFN1, and MFN2) were detected. $\alpha$-tubulin was used as a loading control. D) UCA1 silencing markedly increased the apoptosis in PDAC cells, while mitochondrial fission inhibitor Mdivil reversed this influence. 
activation form of Drp1 (phosphorylated-Drp1 (Ser616)), appeared after UCA1 knockdown, compared with the mock groups (Figure 5C). However, there was no significant change in MFN1 and MFN2 (Figure 5C). Additionally, an application of the mitochondrial fission inhibitor Mdivil reversed the effects of UCA1 knockdown on cell apoptosis in PDAC (Figure 5D). These results suggested that UCA1-driven change in mitochondrial dynamics is coupled with the mitochondrial apoptotic pathway in PDAC.

\section{Discussion}

Accumulating studies have made efforts to uncover the mechanisms oflncRNAs in tumorigenesis of various cancers. Increasing evidence has shown that dysregulated lncRNAs expression contributed to cell proliferation and transformation of malignancies [16, 17]. Urothelial carcinoma associated 1 (UCA1), located on chromosome 19p-13.12, was initially found overexpressed in urinary bladder cancer tissues enhancing the tumorigenicity and invasive potential [18]. There is abundant evidence that has evaluated UCA1 expression in cancer samples obtained from various types of cancer patients. Most research reported that upregulated UCA1 expression existed in tumor samples compared with that in non-tumor samples of the same origin. Generally, the poor prognosis was presented in these patients with overexpression of this lncRNA, which could be recognized as a prognostic factor for overall survival, such as, gastric cancer [19], esophageal cancer [20], hepatocellular carcinoma [21], colon cancer [19], lung cancer [22], ovarian cancer [23], and osteosarcoma [24]. To date, several UCA1-miRNA-mRNA trios have been identified as the main mechanism to promote tumorigenesis. For example, in prostate cancer, UCA1 functions as a microRNA-204 (miR-204) sponge to upregulate CXCR4 expression [25]. In colorectal cancer, UCA1 promotes tumor malignancy via the miR-143/MYO6 axis [26]. In addition, UCA1 has been reported to be implicated in the resistance to chemotherapy [27]. UCA1 plays a critical role in retinoblastoma chemoresistance through downregulating miR-513a-5p [28]. In gastric cancer, UCA1 promoted the cisplatin resistance by recruiting EZH2 and activating the PI3K/AKT pathway to modulate cell apoptosis [29]. So, in PDAC, how does the expression pattern of UCA1 change and what role does it play.

Previous studies had shown that upregulated UCA1 exerted an oncogenic role on tumorigenesis via regulating the Hippo pathway [9] or targeting miR-135a [30]. In addition, increased UCA1 expression could enhance stem cell properties of PDAC cells via the UCA1/KRAS axis [31]. Here, we also revealed that UCA1 is upregulated and involved in cell growth. What's more, data mining showed that an unsatisfactory outcome was presented in patients with a higher UCA1 expression than those with a lower expression. All of this implied that UCA1 worked as a promoter in PDAC progression. However, more studies should be designed for detecting the UCA1 level in the different patient cohorts.

Following, the GSEA analysis showed that high UCA1 expression was positively correlated with cell apoptosis in PDAC. Meanwhile, the flow cytometry and the caspase $3 / 9$ activity detection confirmed the inhibition effect of UCA1 on the cell apoptosis in PDAC cells. Previous studies displayed that UCA1 is involved in regulating the Warburg effect in bladder cancer [10]. Moreover, UCA1 could enhance mitochondrial function through mediating the copy number of mtDNA, ATP production, and mitochondrial membrane potential in bladder cancer [11]. Inspired by this, we speculated that the upregulated UCA1 might be involved in the mitochondrial apoptotic pathway. As a result, we found that UCA1 knockdown led to a significant increase of pro-apoptotic proteins, as well as resulted in a marked increase of cytochrome $\mathrm{c}$ release into cytosolic fractions, which were concordant with our presumption.

In view of this, curiosity drove us to clarify how is UCA1 involved in the mitochondrial apoptotic pathway. Accumulating evidence manifested that the destruction of the dynamic equilibrium of mitochondrial dynamics could adjust mitochondrial reprogramming and resist apoptosis in cancer $[32,33]$. And the idea of mitochondria fragmentation during apoptosis is widely accepted. Previous studies revealed that ablation of Drp1 could inhibit cytochrome c release and apoptosis [34, 35]. Similar to our result, the UCA1 knockdown enhanced the mitochondria fragmentation, as well as increased the expression of Drp1 and Fis1, which would promote mitochondria fragment in turn. These phenomena suggested that UCA1-driven change of mitochondria fragmentation resulted in cytochrome $c$ release from mitochondria. Moreover, a growing volume of studies showed that UCA1 could act as competing endogenous RNAs (ceRNAs) or molecular sponges with microRNAs (miRNAs) to play a post-transcriptional regulatory role in gene expression [36]. However, the aspect of UCA1-miRNA-mRNA interaction in PDAC remains largely unknown. UCA1 enhanced phospho-KRAS expression by interacting with hnRNPA2B1 to regulate stem cell properties in PDAC [31]. A positive regulatory loop was formed, in which UCA1 increased the localization and stability of Yap in the nucleus, and YAP promotes UCA1 expression in PDAC [9]. Hence, further study needs to be performed to clarify how UCA1 mediates the mitochondrial morphology, whether it mediated the expression of Drp1 or the localization and stability of Drp1 in mitochondria.

Taken together, we provided the first evidence that UCA1-driven change in the mitochondrial dynamics is coupled to the mitochondrial apoptotic pathway in PDAC. These findings provide a deep understanding of the potential mechanism of UCA1 in the development of PDAC. And, UCA1 has the potential to serve as a candidate biomarker for prognosis and a target for new PDAC therapies. 
Acknowledgments: This research was supported by the Youth Talent Fund of Lianyungang First People’s Hospital (QN1904).

\section{References}

[1] POLIREDDY K, CHEN Q. Cancer of the Pancreas: Molecular Pathways and Current Advancement in Treatment. J Cancer 2016; 7: 1497-1514. https://doi.org/10.7150/jca.14922

[2] MOHAMMED S, VAN BUREN G 2nd, FISHER WE. Pancreatic cancer: advances in treatment. World J Gastroenterol 2014; 20: 9354-9360. https://doi.org/10.3748/wjg.v20. i28.9354

[3] MERCER TR, DINGER ME, MATTICK JS. Long non-coding RNAs: insights into functions. Nat Rev Genet 2009; 10: 155-159. https://doi.org/10.1038/nrg2521

[4] MERCER TR, MATTICK JS. Structure and function of long noncoding RNAs in epigenetic regulation. Nat Struct Mol Biol 2013; 20: 300-307. https://doi.org/10.1038/nsmb.2480

[5] DIMOND A, FRASER P. Molecular biology. Long noncoding RNAs Xist in three dimensions. Science 2013; 341: 720 721. https://doi.org/10.1126/science. 1243257

[6] DENZLER R, AGARWAL V, STEFANO J, BARTEL DP, STOFFEL M. Assessing the ceRNA hypothesis with quantitative measurements of miRNA and target abundance. Mol Cell 2014; 54: 766-776. https://doi.org/10.1016/j.molcel.2014.03.045

[7] SHI X, SUN M, LIU H, YAO Y, SONG Y. Long non-coding RNAs: a new frontier in the study of human diseases. Cancer Lett 2013; 339: 159-166. https://doi.org/10.1016/j.canlet.2013.06.013

[8] XIAO C, WU CH, HU HZ. LncRNA UCA1 promotes epithelial-mesenchymal transition (EMT) of breast cancer cells via enhancing Wnt/beta-catenin signaling pathway. Eur Rev Med Pharmacol Sci 2016; 20: 2819-2824.

[9] ZHANG M, ZHAO Y, ZHANG Y, WANG D, GU S et al. LncRNA UCA1 promotes migration and invasion in pancreatic cancer cells via the Hippo pathway. Biochim Biophys Acta Mol Basis Dis 2018; 1864: 1770-1782. https://doi. org/10.1016/j.bbadis.2018.03.005

[10] LI Z, LI X, WU S, XUE M, CHEN W. Long non-coding RNA UCA1 promotes glycolysis by upregulating hexokinase 2 through the mTOR-STAT3/microRNA143 pathway. Cancer Sci 2014; 105: 951-955. https://doi.org/10.1111/cas.12461

[11] LI HJ, SUN XM, LI ZK, YIN QW, PANG H et al. LncRNA UCA1 Promotes Mitochondrial Function of Bladder Cancer via the MiR-195/ARL2 Signaling Pathway. Cell Physiol Biochem 2017; 43: 2548-2561. https://doi. org/10.1159/000484507

[12] CHAKRABORTY PK, MUSTAFI SB, XIONG X, DWIVEDI SKD, NESIN V et al. MICU1 drives glycolysis and chemoresistance in ovarian cancer. Nat Commun 2017; 8: 14634. https://doi.org/10.1038/ncomms14634

[13] WANG XM, YAO M, LIU SX, HAO J, LIU QJ et al. Interplay between the Notch and PI3K/Akt pathways in high glucose-induced podocyte apoptosis. Am J Physiol Renal Physiol 2014; 306: F205-213. https://doi.org/10.1152/ajprenal.90005.2013
[14] KOOPMAN WJ, DISTELMAIER F, ESSELING JJ, SMEITINK JA, WILLEMS PH. Computer-assisted live cell analysis of mitochondrial membrane potential, morphology and calcium handling. Methods 2008; 46: 304-311. https://doi. org/10.1016/j.ymeth.2008.09.018

[15] SHERIDAN C, DELIVANI P, CULLEN SP, MARTIN SJ. Bax- or Bak-induced mitochondrial fission can be uncoupled from cytochrome C release. Mol Cell 2008; 31: 570-585. https://doi.org/10.1016/j.molcel.2008.08.002

[16] PRENSNER JR, CHINNAIYAN AM. The emergence of lncRNAs in cancer biology. Cancer Discov 2011; 1: 391-407. https://doi.org/10.1158/2159-8290.CD-11-0209

[17] GIBB EA, BROWN CJ, LAM WL. The functional role of long non-coding RNA in human carcinomas. Mol Cancer 2011; 10: 38. https://doi.org/10.1186/1476-4598-10-38

[18] WANG F, LI X, XIE X, ZHAO L, CHEN W. UCA1, a nonprotein-coding RNA up-regulated in bladder carcinoma and embryo, influencing cell growth and promoting invasion. FEBS Lett 2008; 582: 1919-1927. https://doi.org/10.1016/j. febslet.2008.05.012

[19] ZHENG Q, WU F, DAI WY, ZHENG DC, ZHENG C et al. Aberrant expression of UCA1 in gastric cancer and its clinical significance. Clin Transl Oncol 2015; 17: 640-646. https://doi.org/10.1007/s12094-015-1290-2

[20] LI JY, MA X, ZHANG CB. Overexpression of long non-coding RNA UCA1 predicts a poor prognosis in patients with esophageal squamous cell carcinoma. Int J Clin Exp Pathol 2014; 7: 7938-7944.

[21] ZHENG ZK, PANG C, YANG Y, DUAN Q, ZHANG J et al. Serum long noncoding RNA urothelial carcinoma-associated 1: A novel biomarker for diagnosis and prognosis of hepatocellular carcinoma. J Int Med Res 2018; 46: 348-356. https://doi.org/10.1177/0300060517726441

[22] WANG HM, LU JH, CHEN WY, GU AQ. Upregulated lncRNA-UCA1 contributes to progression of lung cancer and is closely related to clinical diagnosis as a predictive biomarker in plasma. Int J Clin Exp Med 2015; 8: 1182411830 .

[23] BIAN Z, JIN L, ZHANG J, YIN Y, QUAN C et al. LncRNAUCA1 enhances cell proliferation and 5-fluorouracil resistance in colorectal cancer by inhibiting miR-204-5p. Sci Rep 2016; 6: 23892. https://doi.org/10.1038/srep23892

[24] WEN JJ, MA YD, YANG GS, WANG GM. Analysis of circulating long non-coding RNA UCA1 as potential biomarkers for diagnosis and prognosis of osteosarcoma. Eur Rev Med Pharmacol Sci 2017; 21: 498-503.

[25] HE C, LU X, YANG F, QIN L, GUO Z et al. LncRNA UCA1 acts as a sponge of miR-204 to up-regulate CXCR4 expression and promote prostate cancer progression. Biosci Rep 2019; 39: BSR20181465. https://doi.org/10.1042/BSR20181465

[26] LUAN Y, LI X, LUAN Y, ZHAO R, LI Y et al. Circulating lncRNA UCA1 Promotes Malignancy of Colorectal Cancer via the miR-143/MYO6 Axis. Mol Ther Nucleic Acids 2020; 19: 790-803. https://doi.org/10.1016/j.omtn.2019.12.009

[27] WANG H, GUAN Z, HE K, QIAN J, CAO J et al. LncRNA UCA1 in anti-cancer drug resistance. Oncotarget 2017; 8: 64638-64650. https://doi.org/10.18632/oncotarget.18344 
[28] YANG L, ZHANG L, LU L, WANG Y. IncRNA UCA1 Increases Proliferation and Multidrug Resistance of Retinoblastoma Cells Through Downregulating miR-513a-5p. DNA Cell Biol 2020; 39: 69-77. https://doi.org/10.1089/ dna.2019.5063

[29] DAI Q, ZHANG T, PAN J, LI C. LncRNA UCA1 promotes cisplatin resistance in gastric cancer via recruiting EZH2 and activating PI3K/AKT pathway. J Cancer 2020; 11: 38823892. https://doi.org/10.7150/jca.43446

[30] ZHANG X, GAO F, ZHOU L, WANG H, SHI G et al. UCA1 Regulates the Growth and Metastasis of Pancreatic Cancer by Sponging miR-135a. Oncol Res 2017; 25: 1529-1541. Oncol Res 2017; 25: 1529-1541.

[31] LIU Y, FENG W, GU S, WANG H, ZHANG Y et al. The UCA1/KRAS axis promotes human pancreatic ductal adenocarcinoma stem cell properties and tumor growth. Am J Cancer Res 2019; 9: 496-510.

[32] YOULE RJ, VAN DER BLIEK AM. Mitochondrial fission, fusion, and stress. Science 2012; 337: 1062-1065. https://doi. org/10.1126/science. 1219855
[33] SENFT D, RONAI ZA. Regulators of mitochondrial dynamics in cancer. Curr Opin Cell Biol 2016; 39: 43-52. https:// doi.org/10.1016/j.ceb.2016.02.001

[34] BRECKENRIDGE DG, STOJANOVIC M, MARCELLUS RC, SHORE GC. Caspase cleavage product of BAP31 induces mitochondrial fission through endoplasmic reticulum calcium signals, enhancing cytochrome c release to the cytosol. J Cell Biol 2003; 160: 1115-1127. https://doi.org/10.1083/ jcb.200212059

[35] BROOKS C, WEI Q, FENG L, DONG G, TAO Y et al. Bak regulates mitochondrial morphology and pathology during apoptosis by interacting with mitofusins. Proc Natl Acad Sci U S A 2007; 104: 11649-11654. https://doi.org/10.1073/ pnas.0703976104

[36] LIN C, WANG Y, WANG Y, ZHANG S, YU L et al. Transcriptional and posttranscriptional regulation of HOXA13 by lncRNA HOTTIP facilitates tumorigenesis and metastasis in esophageal squamous carcinoma cells. Oncogene 2017; 36: 5392-5406. https://doi.org/10.1038/onc.2017.133 